\title{
INTRODUCTION
}

\section{Exploring the Universe from Argentina}

In this publication the four major international projects in astrophysics and cosmology being developed in Argentina are described: QUBIC and LLAMA in the Puna of the province of Salta, the Pierre Auger Observatory and ANDES in the region of Cuyo.

The Pierre Auger Observatory is the largest observatory of cosmic rays in the world developed by a collaboration of about 400 scientists, engineers and technicians from 17 countries. The construction of the Observatory in the Departments of Malargüe and San Rafael of the Province of Mendoza, on a flat area at 1340-1610m above sea level spanning a surface of thousands square kilometers was concluded in 2008. Its purpose is the study of cosmic rays that are massive, charged particles coming from beyond the solar system. Of topical interest in Particle Physics and High Energy Astrophysics are the cosmic rays of the highest known energies, up to few times $10^{20}$ electron-volts, nearly one hundred million times the highest energies produced so far by the most powerful accelerator on Earth. The ultimate origin and the full detailed properties of those ultra-high energy particles remain open questions. Cosmic rays with energies greater than few times $10^{20}$ electron volts have not been observed, and it is not known whether this is due to suppression in the propagation of cosmic rays in the intergalactic medium, or if it is due to an exhaustion of the injection power of astrophysical sources in nature. It is expected that an answer of this fundamental question will be found by the observatory's current upgrade called AugerPrime. It began in 2015, with a planned complete installation in 2022, and will operate for over a decade. The protagonist role of Argentine institutions, scientists, engineers and technicians in the successful realization of this project, provides our country with an excellent international reputation and is an example to follow to host future international projects.

ANDES (Agua Negra Deep Experiment Site) is a Deep Underground Laboratory (DUL) project, foreseen as an international laboratory to be constructed in the planned Agua Negra tunnel that will connect the San Juan Argentine province to the Coquimbo province in Chile. The main scientific motivation is to find the properties of dark matter and neutrinos. Dark matter is thought to account for approximately $85 \%$ of the matter in the Universe, and it is believed to be composed of particles that do not absorb, reflect, or emit light, so dark matter cannot be detected by electromagnetic radiation. Neutrinos are elusive subatomic particles that zip through the cosmos at nearly the speed of light and that only interact via the weak subatomic force and gravity. To avoid the intense background of penetrating cosmic rays that makes difficult the detection of the weakly interacting dark matter and neutrino particles, DULs that use a great overload of rock as natural protection have been constructed in the Northern Hemisphere. ANDES would be the first DUL to be constructed in the Southern Hemisphere with more than $1000 \mathrm{~m}$ of overburden. ANDES will be important to study the transformation of one neutrino species (flavor) into another one in media with varying mass density, which may lead to the explanation of the cosmological dominance of matter over antimatter in the present Universe. Furthermore, a DUL in the Southern Hemisphere will be necessary to know whether a tentative detection of dark matter signal modulation is due to the movement of the Earth around the Sun or to possible seasonal atmospheric effects. Besides contributions to fundamental physics, astrophysics and cosmology, ANDES will allow unique experimental possibilities for a diversity of other disciplines.

LLAMA (Large Latin American Millimeter Array). In the 1980's, new horizons for ground-based astrophysics and cosmology by the observation of the Universe at millimeter wavelengths were open thanks to the development of new technological capabilities. To take advantage of those new capabilities, in the 1990's a preliminarily search began in the provinces of Salta and Jujuy for the dry and high-altitude sites that are needed for sub-millimeter/millimeter observations. To install an antenna for sub-millimeter/millimeter observations (LLAMA), such early searches were followed systematically during several years by meteorological and topographical campaigns in different locations of the Puna by the Argentine Institute for Radio Astronomy (IAR), with instrumentation lent by the National Autonomous University of México (UNAM). Two potential sites were identified. To comply with the required atmospheric and logistic conditions for the installation of the LLAMA antenna, it was finally chosen a site called Alto Chorrillos at $4832 \mathrm{~m}$ altitude, about 18 $\mathrm{km}$ in straight line from the small town of San Antonio de los Cobres, in the Puna of the province of Salta. 
LLAMA is a binational collaboration to install a millimeter antenna of $12 \mathrm{~m}$ diameter in Alto Chorrillos. After site selection, members of IAR and the University of Sao Paulo (USP) worked on different aspects of project planning and management. The Brazilian Foundation FAPESP bought the antenna with a grant of USD9 million in 2012, and in 2014 an agreement was signed between the Argentine ministry of science and technology MinCyT, FAPESP and USP. At 180 $\mathrm{km}$ from the Atacama Large Millimeter Array (ALMA), the interferometric connection of LLAMA with that array will allow to increase by an order of magnitude the angular resolution of the sources, enhancing the scientific return of the observations. As an additional antenna in the Event Horizon Telescope (EHT), LLAMA will be able to participate in global astrophysical projects, as the one that recently produced the first image of the shadow of a supermassive black hole. Therefore, at a relative low cost compared with the fees to become member country of ALMA (which had an initial construction cost of USD1.4 billon), LLAMA will allow the participation of Argentine and Brazilian scientists in international projects of front-line research. The LLAMA antenna is a multipurpose instrument that will also operate as a stand-alone telescope making contributions in solar and planetary sciences, astrophysics, and cosmology. Presently, all components of the antenna are on site, but it should be assembled with the participation of the staff that will be in charge of the future operations.

QUBIC (Q\&U Bolometric Interferometer for Cosmology) is being built by an international collaboration between laboratories in France, Italy, UK, Ireland, USA and Argentina. It will be deployed in Alto Chorrillos at about 400m from LLAMA. The scientific motivation of QUBIC is to find in the polarization of the cosmic microwave background (CMB) the signature of primordial gravitational waves generated by a very early accelerated expansion of the Universe. That cosmic inflationary phase is the current paradigm that accounts for the main large-scale properties of the Universe. So far polarizations of the CMB in the first order E modes due to density fluctuations, and in the secondary B modes at small angular scales (due to weak gravitational lensing) have been observed. However, primordial B-modes due to gravitational waves produced during the inflation epoch that stretched quantum fluctuations by a factor larger than $10^{30}$ to expected angular scales of about one degree, have not been observed. The observation of primordial B-modes is a great experimental challenge, due to their likely tiny amplitude, and because their signature can be contaminated with polarized foregrounds of galactic origin, as has been the case in a reported tentative detection of B-mode polarization. Currently, there are several experimental efforts pursuing the search for primordial B-modes. QUBIC will join them with a novel control of systematic instrumental effects, and improved subtraction methods of astrophysical foregrounds, by interferometry with bolometers of high sensitivity. The successful performance of QUBIC will open a new road to search for the elusive primordial B-modes of CMB polarization, footprints of gravitational waves from an inflationary period during the earliest moments in the history of the Universe.

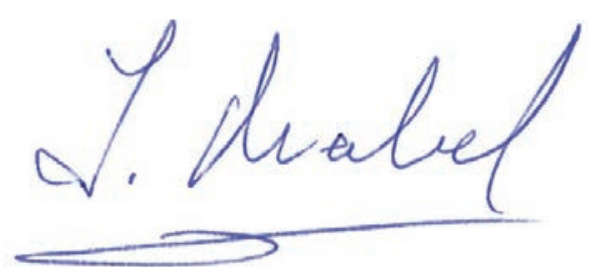

Félix Mirabel

Bio

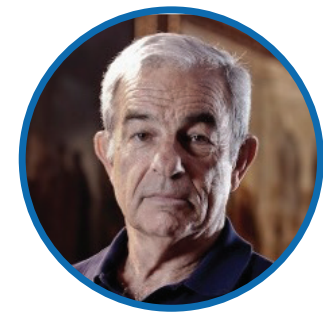

Félix Mirabel

Félix Mirabel proposed in December 2007 to the Ministry of Science and Technology of Argentina the idea of the project LLAMA described in this publication, initiating the steps for the financial participation of Brazil in this project. As scientific researcher, Mirabel has been principal driver in the discoveries of three new classes of cosmic objects: Microquasars, which are objects of topical interest in High Energy Astrophysics, and Ultraluminous Infrared Galaxies, and Tidal Dwarf Galaxies. In the last decade, Mirabel has concentrated his research in Cosmology, working on the role of astrophysical high energy sources at cosmic dawn. Because of his scientific contributions he received several distinctions in Europe, USA and Argentina: The Doctorate Honoris Causa from the University of Barcelona, the Prize on Scientific Excellence of the French Atomic Energy Commission, the "Grand Prize Deslandes" of the French Academy of Sciences, the Rossi Prize of the High Energy Division of the American Astronomical Society, the EPSCOR Prize for 
Science Productivity of the National Science Foundation of USA, and the Guggenheim fellowship to finance his research at Caltech. In Argentina he received the Houssay Prize of MinCyT for the Trajectory, the Konex foundation Prize for research in Physics and Astronomy in the 20002010 decade, and the Dr. Sahade Prize of the Argentine Astronomical Association in 2018. Félix Mirabel was born in Uruguay and is a naturalized citizen of Argentina. After finishing high school in Montevideo, obtained a Ph.D. in Astronomy at the University of La Plata, and the title of Professor of Philosophy from the University of Buenos Aires. In March 1976, he leaves to England as a post-doc fellow at the Jodrell Bank Radio Observatory, financed by fellowships of the British Council and the University of Manchester. Two years later he moved to the USA carrying out research at several institutions: as post-doc at the University of Maryland, as associate researcher at Caltech, and as Professor of Physics at the University of Puerto Rico with research at the Arecibo Radio Observatory. Before his return to Argentina, he moved back to Europe becoming Director of Research at the French Atomic Commission, from where he was later commissioned as Representative and Director of the Office of Science in Chile of the European Southern Observatories (ESO). At present, Mirabel is emeritus Researcher of the National Research Council of Argentina at the Institute of Astronomy and Space Physics in Buenos Aires, and member of the National Academy of Exact, Physical and Natural Sciences of Argentina.

\section{Guest Editor of this Issue}

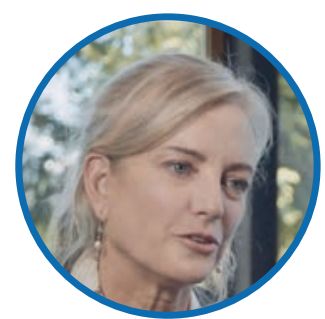

\section{Karen Hallberg}

After finishing high school in the province of Jujuy, Argentina, Karen Hallberg studied two years of electronic engineering at the National University of Rosario and later obtained a full scholarship to study physics at the Instituto Balseiro in Bariloche (National Atomic Energy Commission CNEA, and National University of Cuyo), where she also did her PhD in Physics. She had postdoctoral positions at the Max-Planck Institutes in Stuttgart and in Dresden, Germany. Currently, she is Principal Researcher of the Argentine Council for Science and Technology (CONICET) working at the Bariloche Atomic Center (CAB) and is Professor at the Balseiro Institute. She was recently elected as International Councilor of the American Physical Society (APS) and invited as a member of the World Economic Forum's Global Future Council on Quantum Applications. She is also a Council member of the Pugwash Conferences for Science and World Affairs (1995 Peace Nobel Prize), member of the Argentine Committee for Ethics in Science and Technology, corresponding member of the Argentine Academy for Exact, Physical and Natural Sciences, member of the Latin American Academy of Sciences, Senior Associate of the ICTP (International Center for Theoretical Physics, Trieste), Associate Member ICTPSAIFR, member of the International Advisory Council of the International Institute of Physics, Natal (Brazil) and member of the Editorial Board of Physical Review Research (APS). She served as the Head of the Condensed Matter Department at CAB, as the Argentine representative to the Latin American Center of Physics (CLAF), as member of the Board of Directors of the Aspen Science Center, and on several other national and international committees. She was awarded the 2019 L'Oreal-UNESCO International Award For Women in Science (for Latin America), honorary doctorates at the National University of Jujuy and at the University Siglo XXI, an honorary IAEA Marie Sklodowska Curie Fellowship, the 2005 Guggenheim Fellowship and was an Aspen Ideas Festival Scholar. Her research topics include the development of state-of-the-art computational approaches to understand the physics of quantum matter and nanoscopic systems. She is actively committed to reducing the gender gap and increasing awareness of ethical considerations in science. 